\title{
Repair of the Injured Spinal Cord by Implantation of a Synthetic Degradable Block Copolymer in Rat
}

\author{
Vincent PERTICI ${ }^{1}$, Thomas TRIMAILLE ${ }^{2}$, Jérôme LAURIN ${ }^{1}$, Marie-Solenne \\ FELIX $^{1}$, Tanguy MARQUESTE ${ }^{1}$, Brigitte PETTMANN ${ }^{3}$, Jean-Paul \\ CHAUVIN $^{4}$, Didier GIGMES ${ }^{2 * \bowtie}$, Patrick DECHERCHI ${ }^{1 * \bowtie}$ \\ * These authors have shared seniority.
}

${ }^{1}$ Aix-Marseille Université - CNRS

UMR 7287 «Institut des Sciences du Mouvement : Etienne-Jules MAREY »(ISM)

Equipe « Plasticité des Systèmes Nerveux et Musculaire » (PSNM)

Parc Scientifique et Technologique de Luminy

Faculté des Sciences du Sport de Marseille

CC910 - 163 Avenue de Luminy

F-13288 Marseille cedex 09, France

${ }^{2}$ Aix-Marseille Université - CNRS

UMR 7273 « Institut de Chimie Radicalaire » (ICR)

Equipe « Chimie Radicalaire Organique et Polymères de Spécialité » (CROPS)

Case 562 - Avenue Escadrille Normandie-Niemen

13397 Marseille Cedex 20, France.

${ }^{3}$ Aix-Marseille Université - INSERM

UMR_S 901 «Institut de Neurobiologie de la Méditerranée » (INMED)

Equipe « Plasticité Développementale des Synapses GABAergique »

BP13 - 163 Avenue de Luminy

F-13273 Marseille cedex 09, France

${ }^{4}$ Aix-Marseille Université - CNRS

UMR 7288 « Institut de Biologie du Développement de Marseille » (IBDM)

Plateforme « Microscopie Electronique »

CC907 - 163 Avenue de Luminy

F-13288 Marseille cedex 09, France

\section{Corresponding authors:}

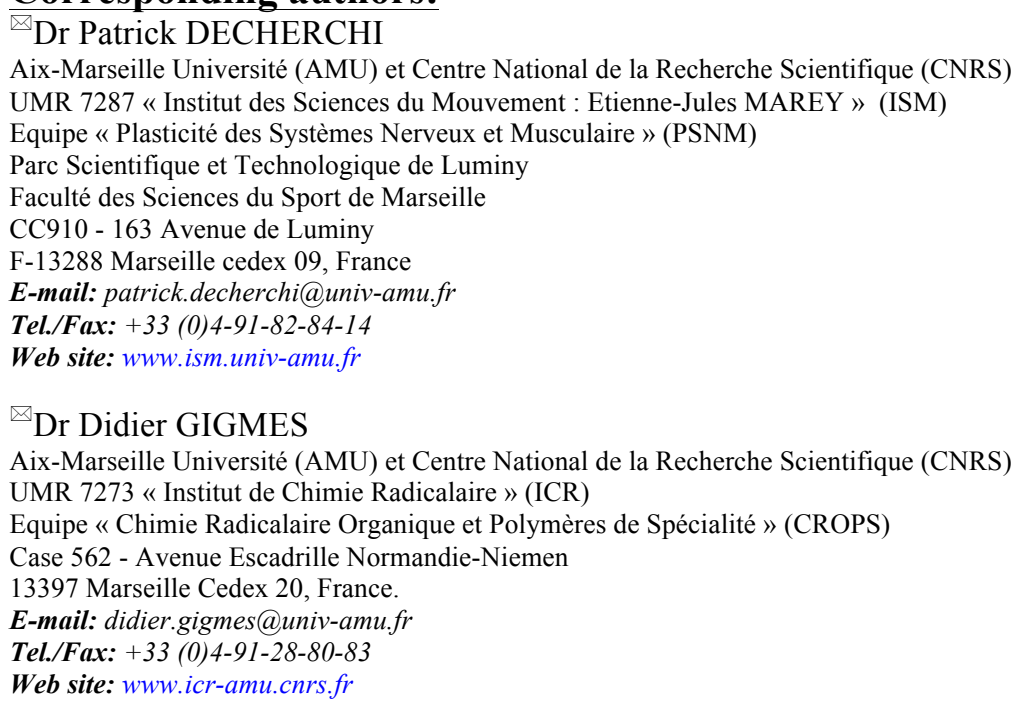




\begin{abstract}
The present study is designed to assess the properties of a new degradable PLA- $b$ PHEMA block copolymer hydrogel and its therapeutic effectiveness after implantation following a thoracic spinal cord hemisection on rats. Degradable characteristics and porous aspect of the scaffold are respectively analyzed by the evaluation of its mass loss and electron microscopy. The biomaterial toxicity is measured through in vitro tests based on motoneuron survival and neurite growth on copolymer substrate. Functional measurements are assessed by the Basso, Beattie and Bresnahan (BBB) and the Dynamic Weight Bearing (DWB) tests during 8 weeks post-surgery. Histological analyses are achieved to evaluate the presence of blood vessels and axons, the density of the glial scar, the inflammatory reaction and the myelination at the lesion site and around it. The results indicate that the synthetic PLA- $b$ PHEMA block copolymer is a non-toxic and degradable biomaterial that provides support for regenerating axons and seems to limit scar tissue formation. Additionally, the implantation of the porous PLA- $b$-PHEMA scaffold enhances locomotor improvement. The observed functional recovery highlights the potential benefits of plain tissue engineering material, which can further be optimized by bioactive molecule functionalization or transplanted cell encapsulation.
\end{abstract}

Keywords: PLA- $b$-PHEMA, Behavioral recovery, Spinal cord hemisection, Axonal regrowth, Weight-bearing distribution. 


\section{Introduction}

The disruption of spinal cord ascending and descending pathways following traumatic injury to the central nervous system (CNS) results in sensory, motor and autonomic dysfunctions below the site of injury. Currently, there are no effective clinical treatments that significantly promote functional recovery in patients with spinal cord injury (SCI) [1]. This failure is mainly attributed to the lack of axonal regrowth. In experimental studies on animals, therapeutic strategies aim at bridging the spinal cord gap and limiting the development of a glial scar by filling the lesion site with various materials such as cells transplant, biomaterials or their combination [2-5].

Biomaterials present several advantages for spinal cord repair due to their structural and chemical versatility [6]. Additionally, biomaterials can be used as a delivery device to release pharmacological treatment and/or encapsulated cells. Although therapeutic effects were observed, no material has currently established itself as a dominant choice for CNS repair [7]. Among those biomaterials, the synthetic polymers poly(lactic acid) (PLA) and poly(2-hydroxyethyl methacrylate) (PHEMA), providing low batch-to-batch variability, have extensively been studied in rat SCI model. Both PLA and PHEMA polymers do not only display specific interesting features but also few drawbacks.

On one hand, PLA cylinders have been used as a temporary extracellular matrix due to their degradable characteristics based on the hydrolysis of the polyester bond [8]. The main advantage of degradable biomaterial is that the polymer scaffold avoids permanent foreign body reaction and continuous inflammatory response [9]. It was demonstrated that PLA scaffolds were not detrimental to severed axons and allowed axonal ingrowth after a complete spinal cord transection in a rat model [10]. Moreover, PLA scaffolds impregnated with brainderived neurotrophic factor (BDNF) or filled with Schwann cells promoted a more rapid axonal regeneration $[11,12]$. However, PLA scaffolds are considered to be rather stiff and brittle materials for being implanted into soft tissue [13]. Moreover, the lack of PLA scaffold wettability hampers the transport of nutrients and fluids through its porous network [14]. 
On the other hand, PHEMA porous hydrogels have been proposed for restoring the anatomical continuity of damaged neural structures. Their high water content and mechanical stability, mimicking soft tissue's extracellular matrix, provided support for axonal regeneration $[15,16]$. However, polymers such as PHEMA present several shortcomings. Indeed, as a non-degradable polymer, hydrogel calcification and prolonged inflammatory response might limit long-term axonal regeneration [7, 17]. Furthermore, axonal regrowth is restricted to the existing pores of the biomaterial.

In order to benefit from the suitable complementary properties of both polymers, our strategy relied on the synthesis of a PLA- $b$-PHEMA block copolymer, based on the combination of ring opening polymerization (ROP) and nitroxide mediated polymerization (NMP) techniques [18]. By combining PLA and PHEMA, the resulting copolymer can indeed display desired properties such as bioresorbability, fitting stiffness, hydrophilicity, cell adhesion and pendant hydroxyl functions, which may be partly exploited for further coupling of suitable bioactive peptides [19]. Such characteristics prompted us to envision the use of this block-copolymer in the frame of spinal cord repair. Thereafter, from this PLA- $b$-PHEMA block copolymer, an original highly porous tridimensional scaffold was prepared by the use of a modified freeze-extraction method and implanted into a hemisected rat spinal cord [20].

Typically, the present study was designed to assess the properties of this di-block copolymer hydrogel and its therapeutic effectiveness after implantation following a thoracic spinal cord hemisection on rats. For that purpose, degradable characteristics and porous aspect of the scaffold were analyzed. The biomaterial toxicity was evaluated through in vitro tests based on motoneuron survival and neurite growth on copolymer substrate. The functional measurements were performed during 8 weeks post-surgery. Improvement of the behavioral function was assessed by: 1) the Basso, Beattie and Bresnahan (BBB) test, a method to evaluate the general locomotor outcomes, and 2) the Dynamic Weight Bearing (DWB) test which assesses static postural control through weight distribution on each paw [21, 22]. Furthermore, histological measurements were carried out to confirm the presence of axons, 
the density of the glial scar and the myelination at and around the lesion site. The 8-week period has previously shown to been sufficient so as to observe numerous therapeutic effects of various biomaterials in spinal cord injury models [16, 23, 24]. Moreover, this time interval matched with the onset of the PLA- $b$-PHEMA mass loss due to its degradation.

\section{Materials and methods}

\subsection{Animals.}

Twenty-eight adult male Sprague Dawley rats, weighting 250-300 g (Centre d'Elevage Roger JANVIER ${ }^{\circledR}$, Le Genest Saint Isle, France), were housed in smooth-bottomed plastic cages at $22{ }^{\circ} \mathrm{C}$ with a $12 \mathrm{~h}$ light/dark cycle. Food (Safe ${ }^{\circledR}$, Augy, France) and water were available ad libitum. An acclimation period of 1 week was allowed before the initiation of the experiment. All animals were weighted before each experiment step.

Animals were assigned to the following treatment groups: 1) the Control group ( $n=7)$ in which no surgery was performed, 2) the SHAM groups $(n=6)$ in which a surgery was performed without injuring the spinal cord, 3$)$ the TH group $(n=7)$ in which the hemisected spinal cord cavity was left empty and 4) the $\mathrm{TH}+$ Copolymer group $(\mathrm{n}=8)$ in which the hemisected spinal cord cavity was immediately implanted with a block of PLA- $b$-PHEMA hydrogel. The 7 and 8 rats of the $\mathrm{TH}$ and $\mathrm{TH}+$ Copolymer groups respectively, whose spinal cord was properly hemisected as proved by cresyl violet staining, were kept for further analyses (behavioral results).

\subsection{Ethical approval.}

Anaesthesia and surgical procedures were performed according to the French law on animal care guidelines and the Animal Care Committees of Aix-Marseille Université (AMU) and Centre National de la Recherche Scientifique (CNRS) approved our protocols. Individual conducting researches were listed in the authorized personnel section of the animal research 
protocol or added to a previously approved protocol (License A 13 01306). Furthermore, experiments were performed following the recommendations provided in the Guide for Care and Use of Laboratory Animals (U.S. Department of Health and Human Services, National Institutes of Health) and in accordance with the European Community's council directive of 24 November 1986 (86/609/ EEC). No clinical sign of pain or unpleasant sensation (i.e. screech, prostration, hyperactivity, anorexia) and no paw-eating behavior were observed through the study.

\subsection{Polymer synthesis and characteristics.}

PLA- $b$-PHEMA block copolymer (13000-7000 g.mol ${ }^{-1}$, i.e., 65/35 wt\% composition) was obtained as previously described by performing the NMP of HEMA from a previously prepared SG1 ( $N$-tert-butyl- $N$-1-diethylphosphono-(2,2-dimethylpropyl))-functionalized PLA macroalkoxyamine (PLA-SG1) (Figure 1A) [18].

The molar mass of the PHEMA block was obtained from ${ }^{1} \mathrm{H}$ NMR spectroscopy on the purified block copolymer after double precipitation in diethyl ether (Figure 1B), based on the known molar mass of the PLA block determined by size exclusion chromatography (SEC) using universal calibration from the Mark-Houwink parameters [18].

\subsubsection{In vitro degradation.}

A PLA- $b$-PHEMA film of $1 \mathrm{~mm}$ thick was prepared by solvent cast from polymer solution in THF (10 wt\%). The solvent was allowed to evaporate overnight and the films were further dried in vacuum oven at $35^{\circ} \mathrm{C}$. The film was then cut into square pieces (roughly $5 \times 5$ x $1 \mathrm{~mm}^{3}$ ) of about $35-40 \mathrm{mg}$ and accurately weighted. Polymer samples were plunged into 2 $\mathrm{mL}$ of $0.1 \mathrm{M}$ phosphate buffer, $\mathrm{pH} 7.4$ and slowly agitated at $37{ }^{\circ} \mathrm{C}$. Degradation media were changed every week to prevent acidification. Sample of eroded polymers collected at predetermined times were rinsed with milli-Q water and dried to constant weight prior to determination of mass loss and averaged molecular weight. The mass loss $(M L \%)$ was 
evaluated by gravimetric analysis and calculated by: $M L \%=100\left(m_{0}-m_{\mathrm{D}}\right) / m_{0}$, where $m_{0}$ is the original dry mass of the sample and $m_{\mathrm{D}}$ the residual dry mass of the sample at degradation time $t .{ }^{1} \mathrm{H}$ NMR analysis of samples at predetermined times in deuterated dimethyl sulfoxide (DMSO-d6) were performed to assess the PLA block degradation.

\subsubsection{Survival and neurite-promoting capacities.}

E14 rat spinal motoneurons were purified as previously described [25]. In brief, cells were dissociated from ventral spinal cord after trypsin treatment. The largest cells were isolated by centrifugation on a $6.5 \%$ metrizamide density gradient. The immunoaffinity purification step usually performed by immunopanning was replaced by a cell-sorting step using magnetic microbeads $[25,26]$. Cells in the metrizamide fraction were incubated with a mouse monoclonal antibody against rat p75NTR (Ig192). Subsequently, motoneurons were incubated with magnetic microbeads conjugated to rat anti-mouse secondary antibodies, thus allowing the purification of motoneurons on separating columns (Miltenyi Biotec, Inc.). After a final centrifugation through a BSA cushion, motoneurons were resuspended in Neurobasal medium (Life Technologies, SAS, Saint Aubin, France). Neurobasal medium was supplemented with $2 \%$ (vol/vol) horse serum, $25 \mu \mathrm{M}$ L-glutamate, $50 \mu \mathrm{M} \beta$-mercaptoethanol, $0.5 \mathrm{mM}$ L-glutamine, and 2\% (vol/vol) B-27 supplement (Life Technologies, SAS). The different neurotrophic factors were used at the optimal concentrations as follows: BDNF (R\&D Systems Europe, Inc., Lille, France) at 1 ng.ml ${ }^{-1}$, rat glial cell line-derived neurotrophic factor (GDNF) at $100 \mathrm{pg} \cdot \mathrm{ml}^{-1}$ and rat ciliary neurotrophic factor (CNTF) at 10 ng. $\mathrm{ml}^{-1}$ (Sigma Chemical Co., Perth WA, Australia). All factors were added at the time of cell seeding. Cells were plated in 4-well dishes (Nunc) containing $12 \mathrm{~mm}$ diameter glass coverslips previously treated with either polyornithine/laminin as described or copolymer substrate prepared by spin-coating from copolymer solution at $2 \mathrm{wt} \%$ in tetrahydrofuran (THF) at $2000 \mathrm{rpm}$, and dried in a vacuum oven at $35^{\circ} \mathrm{C}$ for $48 \mathrm{~h}$ [25]. Reference glass coverslips from spin coating with pure THF (without copolymer) were used as negative 
control. Pictures were taken at 1 and 7 days respectively on a phase-contrast IX71 Olympus microscope.

\subsection{Preparation of porous scaffold.}

The porous PLA- $b$-PHEMA scaffold was prepared through the previously described freeze-extraction method [20]. The PLA- $b$-PHEMA was dissolved in dimethyl sulfoxide (DMSO) to form a $20 \mathrm{wt} \%$ polymer solution. The polymer solution was placed in a mold and frozen at $-20^{\circ} \mathrm{C}$. The frozen polymer solution was immersed in a large volume of distilled water pre-cooled to $4^{\circ} \mathrm{C}$. Due to miscibility between the DMSO and the distilled water, the solvent was extracted out and replaced by distilled water, a non-solvent for PLA- $b$-PHEMA.

${ }^{1} \mathrm{H}-\mathrm{NMR}$ analysis in deuterated chloroform $\left(\mathrm{CDCl}_{3}\right)$ was performed as a control on the dehydrated scaffold (several days under fume hood) and showed no signs of PLA segment degradation. No residual DMSO was detected, to the sensitivity limits of this method.

\subsubsection{Porosity of the sponge-like PLA-b-PHEMA scaffold.}

After having infused the sponge-like PLA- $b$-PHEMA for 2 hours at room temperature in a $0.1 \mathrm{M}$ phosphate buffer $(\mathrm{pH} 7.4)$ containing $2.3 \mathrm{M}$ sucrose, ultrathin sections $(80 \mathrm{~nm})$ were obtained at $-95^{\circ} \mathrm{C}$ using an Ultramicrotome EM UC7 (Leica, Microsystems SAS, Nanterre, France) equipped with a diamond knife (Drukker International, Cuijk, The Netherlands). Sections were collected on a copper grid and stained with uranyl (2\%). Observations were performed on an EM 912 electron microscope (Carl Zeiss SAS, Marly le Roi, France) at a 100-kV accelerating voltage equipped with a BioScan model 792 camera (Gatan France, Evry, France).

\subsection{Surgical protocol.}

Rats were anaesthetized with a choral hydrate intraperitoneal injection $\left(0.5 \mathrm{~g} . \mathrm{kg}^{-1}\right.$, Sigma, St. Louis, MO, USA). The animal's back was shaved and aseptically prepared with a 
povidone-iodine scrub swab stick (Professional Disposables, Orangeburg, NY, USA). Central temperature was maintained constant at $37-38^{\circ} \mathrm{C}$ with a homeothermic blanket (Harvard Apparatus $^{\circledR}$, Holliston, USA) driven by a rectal thermal probe. Rats were then positioned in ventral decubitus and a midline incision was made over the $\mathrm{C}_{6}-\mathrm{T}_{13}$ spinous processes with the aid of a dissecting microscope. Dorsal muscles were cut and maintained on the side using retractors. While firmly maintaining the spinal column by spinous processes, a laminectomy at the $\mathrm{T}_{9}-\mathrm{T}_{10}$ vertebral levels was performed using a Friedman-Pearson Rongeur $(0.7 \mathrm{~mm}$ Cup Curved, FST, Heidelberg, Germany). Following dura incision, the exposed dorsal surface of the spinal cord was rinsed with cold saline to favour vasoconstriction. Finally, a left spinal cord hemi-transection was realized by the use of a sharp blade. Close examination of the cut edges of the cord confirmed that the surface of the tissue was free of meninges or blood clots.

The PLA- $b$-PHEMA hydrogel implant was sized and adapted to the dimension and shape of the cavity. Apposition of the polymer gel with the lesioned spinal surface was realized with a slight downward pressure using a microspatula and ophthalmic sponges. The surgical site containing the polymer implant was covered with a film of bioabsorbable artificial dural substitute (Seamdura ${ }^{\circledR}$, Gunze Ltd., Codman, Johnson and Johnson Company, Kyoto, Japan) to isolate the gel implant from the overlying muscles and mesenchymal tissues. Muscles and skin were sutured (Vicryl ${ }^{\circledR} 4-0$, Ethicon France, Issy Les Moulineaux, France) in anatomical layers. Animals were kept under heat lamps for 12-24 hours. They were rehydrated with a bolus of saline $(3 \mathrm{ml}$, s.c.) to replace fluid lost during the surgical procedure and received a subcutaneous injection of an antalgic (semi-synthetic opioid, buprenorphine, $\left.0.05 \mathrm{mg} \cdot \mathrm{kg}^{-1}\right)$. Then, they were kept in individual cages and daily observed during the next 8 weeks. They were preventively treated with wide spectrum antibiotic (Oxytetracycline, 400 mg. $1^{-1}$, Sigma-Aldrich, Saint-Quentin Fallavier, France) during one week. Manual bladder expression was performed twice daily until bladder reflex was re-established. 


\subsection{Functional assessment of hind limb recovery.}

Behavioral functions were assessed by the locomotor BBB test and the DWB test during 8 weeks post-surgery. The data were compared to the pre-surgical values.

\subsubsection{BBB test.}

The hindlimbs functional recovery was assessed during overground movement according to the BBB scale [21]. This rating scale evaluates the rat locomotor function on a 22-points scale. The maximal score of 21 points represents normal movement while 0 point means complete paralysis of the hindlimb. This test is based on the normal recovery observed after SCI and involves detailed analysis of movement including 1) the amplitude of hindlimb joints, 2) the weight-bearing capabilities, 3) the coordination of the movement, 4) the dragging and the placement of each paw, 5) the lifting of the tail and 5) the trunk stability.

\subsubsection{DWB test.}

The weight-bearing distribution was assessed by a biometric floor instrumented cage (Dynamic Weight-bearing, Bioseb ${ }^{\circledR}$ Development, Vitrolles, France). This device consisted of a Plexiglas ${ }^{ø}$ enclosure (W22 $\times$ L22 $\times \mathrm{H} 30 \mathrm{~cm}^{3}$ ) with a calibrated weight transducer pad composed of 44 x 44 captors (Captor surface: $10.89 \mathrm{~mm}^{2}$; Captor threshold: $0.1 \mathrm{~g}$; Matrix Sensor 5250 type: /10, TEKSCAN Inc. Boston, MA, USA). The rat was allowed to move freely within the apparatus for 2 trials of 5 min each. The observer identified each paw of the rat as a unique paw using a synchronized video-recording and a scaled map of the stimulated captors. Only stable paw pressures of at least $0.2 \mathrm{sec}$ were kept for further analysis.

\subsubsection{The pressure exerted by paw.}

The pressure exerted by each paw (in grams) was only measured when the four paws were in contact with the biometric floor and then normalized by the total weight of the rat. Ratios distinguishing the forepaws $v s$. hindpaws and the right $v s$. left side were calculated to 
assess the weight-bearing distribution: 1) the sum of the right and the left forepaws (F) was normalized by the sum of the right and the left hindpaws $(\mathrm{H})(\mathrm{F} / \mathrm{H}$ ratio $), 2)$ the left forepaw (LF) was normalized by the right forepaw (RF) (LF/RF ratio), 3) the left hindpaw (LH) was normalized by the right hindpaw $(\mathrm{RH})(\mathrm{LH} / \mathrm{RH}$ ratio).

\subsubsection{Time spent on 3 paws and on 4 paws.}

The time spent on 3 paws and on 4 paws (in seconds) was measured. The time spent on 2 paws referring to the explorative period was discarded for analysis. Given that the software just kept static position of the rat, the recording period could vary between animals. Consequently, the time periods spent on 3 paws and on 4 paws were normalized by the total time spent in static position for each recording.

\subsection{Spinal cord histology.}

Following behavioral tests, all animals were sacrificed under deep anesthesia with pentobarbital (100 mg.kg-1 i.p., CEVA Santé Animale, Libourne, France), then perfused transcardially with $300 \mathrm{ml}$ of $0.1 \mathrm{M}$ ice-cold phosphate buffer (PBS, $\mathrm{pH}$ 7.4) and followed by the same volume of $4 \%$ phosphate-buffered paraformaldehyde (PBS-PFA, $\mathrm{pH} 7.4$ ). A spinal cord segment extending $5 \mathrm{~mm}$ rostral and caudal to the injury site was immediately dissected, post-fixed for $2 \mathrm{~h}$ at $4{ }^{\circ} \mathrm{C}$ in the same fixative buffer and cryoprotected $24 \mathrm{~h}$ at $4^{\circ} \mathrm{C}$ in $30 \%$ sucrose (Sigma-Aldrich). Tissues were then snap-frozen at $-40^{\circ} \mathrm{C}$ for $30 \mathrm{~s}$ in isopentane solution and stored at $-80^{\circ} \mathrm{C}$ until further use.

At the exception of one thoracic spinal cord per group that was cut longitudinally, all the others were cut coronally into $30 \mu \mathrm{m}$ thick sections using a cryostat (Leica, Microsystems SAS). Sections were mounted on glass microscope slides Superfrost Plus (Thermo Fisher Scientific Inc., Waltham, USA) and stored at $-20^{\circ} \mathrm{C}$. Both Control and SHAM groups were used as reference. 
Cresyl violet staining was used to identify the injury. Sections were rinsed in distilled water for $5 \mathrm{~min}$ and incubated $3 \mathrm{~min}$ in a cresyl violet bath. Sections were then dehydrated through a sequence of ethanol baths $(70 \%, 95 \%$, and $100 \%)$. Sections were finally cleaned in Histolemon for 2 min and medium mounted with coverslip using Permount ${ }^{\circledR}$ (Thermo Fisher Scientific Inc.).

Green FluoroMyelin was used to stain myelin. Briefly, sections were washed with PBS and incubated during 30 min with FluoroMyelin (1/200, Molecular Probes, Life Technologies SAS). Negative controls (without FluoroMyelin) were included.

For nuclear staining, sections were washed with PBS, and incubated 5 min with Hoechst (1/1000, Sigma-Aldrich). After a last wash in PBS, slides were rinsed in distilled water and medium mounted with Mowiol $^{\circledR}$ (Calbiochem, Merck Millipore, Merck KGaA, Darmstadt, Germany).

For immunofluorescence studies, the sections were rinsed with PBS, treated with blocking solution of PBS $+0.2 \%$ of Triton X-100 containing $4 \%$ of Normal Goat Serum (NGS) for $45 \mathrm{~min}$ and incubated overnight at $4^{\circ} \mathrm{C}$ in the same solution with following primary antibodies: mouse anti-neurofilament heavy (NF-H; 1/400, Sigma-Aldrich) for axons, goat anti-glial fibrillary acidic protein (GFAP; 1/1000, Abcam ${ }^{\circledR}$, Paris France) for astrocytes, mouse anti-ionized calcium binding adaptor molecule (Iba-1; 1/100, Abcam ${ }^{\circledR}$ ) for microglia and macrophages and rabbit anti-von Willebrand factor (vWF; 1/100, Abcam ${ }^{\circledR}$ ) for endothelial cells. After treatment with primary antibodies, the sections were washed with PBS and incubated in a solution of PBS containing 4\% of NGS with secondary antibodies: Alexa Fluor $^{\circledR} 488$ donkey anti-goat, Alexa Fluor ${ }^{\circledR} 568$ donkey anti-mouse and Alexa Fluor ${ }^{\circledR} 488$ donkey anti-rabbit $\left(1 / 400\right.$, Invitrogen ${ }^{\circledR}$, Life Technologies SAS). Negative controls (omission of the primary antibodies) were included and used as a reference for all further analysis. Images were taken using Apotome (Carl Zeiss SAS) equipped with a 10x and 40x lens and with AxioVision software (Carl Zeiss SAS). 


\subsection{Statistical analysis.}

Statistical analysis was performed using SigmaStat software program (SigmaStat ${ }^{\circledR}$ 2.03, Statistical software, San Jose, CA, USA). All data are presented as Mean \pm SEM and were compared by ANOVA tests. Post-hoc comparisons were performed with StudentNewman-Keuls multiple post-test comparisons. Results were considered statistically significant, highly significant or very highly significant if the p-value fell below $0.05,0.01$ and 0.001 , respectively.

\section{Results}

\subsection{Characteristics of PLA-b-PHEMA copolymer.}

\subsubsection{In vitro degradation.}

The block copolymer maintained a constant mass over the first 55 days. However, from that time on, an increasing mass loss was observed, reaching about $60 \%$ after 97 days, consistent with the escape of most part of the PLA degradation residues, contributing to $65 \%$ of matrix weight (Figure 2A).

During the first 55 days of no mass loss, only random cleavage of PLA backbone esters through hydrolysis occurs, as typically observed for bulk degrading PLA based materials. This phenomenon leads to the formation and the accumulation of oligo lactic acid residues in the material. This was clearly evidenced by the ${ }^{1} \mathrm{H}$ NMR analysis of the degrading sample at 55 days post-degradation, just before the beginning of mass loss (Figure 2A). Indeed, the spectrum showed significant apparition of the proton signals characteristic of the oligo lactic acid $-\mathrm{CH}$ - protons adjacent to the $\mathrm{OH}$ and $\mathrm{COOH}$ end groups ( $\mathrm{f}$ ' and $\mathrm{f}^{\prime \prime}$ ), as compared to the initial intact block copolymer (presenting only - $\mathrm{CH}$ - lactide proton signals (f) of the inside chain, Figure $\mathbf{2 B}$, top and middle spectra). These small residues can finally escape from the material leading to a matrix mainly composed of PHEMA as assessed by ${ }^{1} \mathrm{H}$ NMR at 90 days post-degradation (Figure 2B, bottom spectrum). 


\subsubsection{Survival and neurite-promoting capacities.}

As soon as the first day post-cell seeding, no adhesion was detected on reference coverslips, as observed by the floating cells, in absence of copolymer (i.e. prepared from pure THF) whereas cellular adhesion was apparent on copolymer films. Moreover, distinct axonal growth was present at the first day and persisted for at least 7 days post-cell seeding. The polyornithine/laminin substrate, known to be an excellent support, promoted higher survival and neurite growth than the copolymer films (Figure 3). To ensure that the lack of adhesion observed on the glass substrate was not due to a toxic effect of potential traces of THF solvent, additional refererence coverslips (arising from THF solution) were covered with polyornithine/laminin were evaluated. Similar adhesion to the pure polyornithine/laminin coverslips was obtained which excluded any toxic effect of the potential solvent traces (data not shown).

\subsubsection{Porosity of the sponge-like PLA-b-PHEMA scaffold.}

The electron microscopy, assessing the porosity of the biomaterial, revealed distinct pores of various sizes ranging from nanometers to several micrometers (Figure 4).

\subsection{Functional assessment of hind limb recovery.}

\subsubsection{The BBB test.}

The BBB scores of the Control and SHAM groups were identical and stable throughout the experiment. Pre-surgical values were similar in all groups $(21 \pm 0)$. One week after the lesion, both the BBB scores of the TH $(2.4 \pm 0.9)$ and the $\mathrm{TH}+$ Copolymer groups $(3.9 \pm 1.4)$ significantly decreased $(\mathrm{p}<0.001)$ compared to the pre-surgical values. The BBB scores in both the TH and the TH+Copolymer groups were significantly lower $(p<0.001)$ than the 2 other groups through the protocol. While no difference was observed between the TH group and the $\mathrm{TH}+$ Copolymer group from the pre-surgical week to the $7^{\text {th }}$ week post-surgery, 
the BBB score of the TH+Copolymer group $(15.9 \pm 0.7)$ was higher $(\mathrm{p}<0.05)$ than the one of the TH group (13 \pm 1.9$)$ at the $8^{\text {th }}$ week (Figure 5).

\subsubsection{The dynamic weight-bearing test.}

\subsubsection{The pressure exerted by paw.}

No difference was observed in the Control and the SHAM groups throughout the experiment. Likewise, no difference was found between these 2 groups. Moreover, presurgical pressures exerted by each paw were similar between the different groups.

The pressures exerted by the LF of both the TH and the TH+Copolymer groups were significantly higher $(\mathrm{p}<0.001)$ than the ones of the Control and the SHAM groups.

The pressures exerted by the RF of both the $\mathrm{TH}$ and the $\mathrm{TH}+\mathrm{Copolymer}$ groups were significantly higher than the ones of the Control $(\mathrm{p}<0.001$ for both $)$ and the SHAM $(\mathrm{p}<0.05$ and $\mathrm{p}<0.01$, respectively) groups. More precisely, the pressure exerted by the RF of the $\mathrm{TH}+$ Copolymer group $(27.7 \pm 1.8 \%)$ at the $1^{\text {st }}$ week post-surgery was significantly higher $(\mathrm{p}<0.001)$ than the pre-surgical one $(17.4 \pm 1.3 \%)$.

The pressures exerted by the LH of both the TH and the TH+Copolymer groups were significantly lower $(\mathrm{p}<0.001)$ than in the Control and the SHAM groups. Specifically, the pressures exerted by the LH of the TH group at the $1^{\text {st }}(15.9 \pm 6.2 \%), 2^{\text {nd }}(23.5 \pm 5.6 \%), 3^{\text {rd }}$ $(25.2 \pm 6.3 \%)$ and $4^{\text {th }}(24.9 \pm 3.2 \%)$ weeks post-surgery were lower $(p<0.001 ; p<0.001 ; p<0.01$; $\mathrm{p}<0.05$, respectively) than the pre-surgical value (34.4 $\pm 2.6 \%)$. Similarly, the pressures exerted by the $\mathrm{LH}$ of the $\mathrm{TH}+$ Copolymer group at the $1^{\text {st }}(15.8 \pm 1.9 \%), 2^{\text {nd }}(20.0 \pm 2.3 \%), 3^{\text {rd }}$ $(24.7 \pm 1.3 \%)$ and $4^{\text {th }}(25.7 \pm 1.6 \%)$ weeks post-surgery were lower $(p<0.001 ; p<0.001 ; p<0.01$; $\mathrm{p}<0.05$, respectively) than the pre-surgical value $(33.1 \pm 1.7 \%)$. At the $5^{\text {th }}$ week post-surgery, the pressure exerted by the $\mathrm{LH}$ of the $\mathrm{TH}$ group $(32.5 \pm 1.6 \%)$ was similar to the ones of the Control $(34.2 \pm 1.5 \%)$ and SHAM $(38.0 \pm 1.5 \%)$ groups but higher $(\mathrm{p}<0.05)$ than the pressure exerted by the $\mathrm{TH}+$ Copolymer group $(27.2 \pm 2.1 \%)$. No difference was observed between groups from the $6^{\text {th }}$ week post-surgery to the end of the protocol. 
No difference was observed in the pressure exerted by the RH within and between the different groups (Figure 6).

\subsubsection{Pressure ratios.}

No difference was observed in the Control and the SHAM groups throughout the experiment. Likewise, no difference was found between these 2 groups. Furthermore, presurgical ratios were similar between the 4 studied groups.

The $\mathrm{F} / \mathrm{H}$ ratios of both the $\mathrm{TH}$ and the $\mathrm{TH}+$ Copolymer group were clearly higher $(\mathrm{p}<0.001)$ than in the Control and SHAM groups. More precisely, the $\mathrm{F} / \mathrm{H}$ ratios of the $\mathrm{TH}$ group at the $1^{\text {st }}(1.00 \pm 0.11), 2^{\text {nd }}(0.73 \pm 0.03)$ and $3^{\text {rd }}(0.75 \pm 0.07)$ weeks post-surgery were higher $(\mathrm{p}<0.001 ; \mathrm{p}<0.05 ; \mathrm{p}<0.05$, respectively) than the pre-surgical value $(0.5 \pm 0.05)$. Similarly, the $\mathrm{F} / \mathrm{H}$ ratio of the $\mathrm{TH}+$ Copolymer group at the $1^{\text {st }}$ week $(1.16 \pm 0.13)$ post-surgery was greater $(\mathrm{p}<0.001)$ than the pre-surgical value $(0.59 \pm 0.06)$. From the $5^{\text {th }}$ week post-surgery to the end of the protocol, no difference was observed between the TH group and the two unlesioned groups (Control and SHAM). Likewise, from the $6^{\text {th }}$ week post-surgery to the $8^{\text {th }}$ week no difference was found between the $\mathrm{TH}+\mathrm{Copolymer}$ group and the two unlesioned ones. However, no difference was noticed between the $\mathrm{TH}$ and the $\mathrm{TH}+$ Copolymer groups throughout the experiment.

The LH/RH ratio of the $\mathrm{TH}$ group at the $1^{\text {st }}$ week $(0.50 \pm 0.13)$ post-surgery was significantly lower $(p<0.01)$ than its pre-surgical value $(1.10 \pm 0.06)$. The $\mathrm{LH} / \mathrm{RH}$ ratios of the $\mathrm{TH}+$ Copolymer group at the $1^{\text {st }}$ week $(0.55 \pm 0.09), 2^{\text {nd }}(0.62 \pm 0.09)$ and $3^{\text {rd }}(0.74 \pm 0.06)$ weeks post-surgery were higher $(\mathrm{p}<0.001 ; \mathrm{p}<0.001 ; \mathrm{p}<0.01$, respectively) than the pre-surgical value (1.21 \pm 0.19$)$. At both the $1^{\text {st }}$ and $2^{\text {nd }}$ weeks post-surgery, the $\mathrm{LH} / \mathrm{RH}$ ratios of the $\mathrm{TH}$ group were lower than the Control ( $p<0.01 ; \mathrm{p}<0.05$, respectively) and the SHAM groups $(\mathrm{p}<0.001$; $\mathrm{p}<0.05$, respectively). Similarly, the ones of the $\mathrm{TH}+\mathrm{Copolymer}$ group were lower than the Control $(\mathrm{p}<0.001 ; \mathrm{p}<0.01$, respectively) and the SHAM groups $(\mathrm{p}<0.001 ; \mathrm{p}<0.01$, respectively). However, no difference was observed between the $\mathrm{TH}$ and the $\mathrm{TH}+\mathrm{Copolymer}$ 
groups during the experiment. No difference in the $\mathrm{LH} / \mathrm{RH}$ ratio was found within and between groups from the $3^{\text {rd }}$ week post-surgery to the end of the protocol.

No difference in the LF/RF ratio was found within and between the different groups (Figure 7).

\subsubsection{Time spent on 4 paws.}

No difference in the time spent on 4 paws was observed within and between the different groups (data not shown).

\subsection{Spinal cord histology.}

Negative controls slices with the omission of the primary antibodies indicated no or very weak aspecific staining. Eight weeks after SCI and PLA- $b$-PHEMA scaffold implantation, cellular and molecular changes at the spinal cord level were examined.

\subsubsection{Morphological histology.}

Coronal and longitudinal spinal cord sections stained by cresyl violet revealed that the spinal cords were properly hemisected and that the extent of the injury was similar between the $\mathrm{TH}$ and the $\mathrm{TH}+\mathrm{Copolymer}$ groups. The disruption of the spinal cord continuity for each injured spinal cord was supported by Fluoromyelin staining which demonstrated an absence of myelin due to axotomy of both descending and ascending axons (data not shown). The Hoechst staining in the $\mathrm{TH}+$ Copolymer group demonstrated that numerous cells migrated into the entire scaffold and that the lesional cavity was filled by the biomaterial (Figure 8A-B).

\subsubsection{Immunolabeling.}

The neurofilament staining displayed NF-H positive fibers that were observed at the tissue/hydrogel interface and into the scaffold. The analysis of successive coronal and longitudinal sections indicated that NF-H positive fibers were present throughout the entire 
scaffold. GFAP staining indicated a higher glial reactivity in spared tissue close to the lesion site in both the $\mathrm{TH}$ and the $\mathrm{TH}+$ Copolymer groups compared to the reference groups. However in the $\mathrm{TH}+$ Copolymer group, GFAP staining was not observed within the biomaterial (Figure 8C-F).

As many nuclei were localized in the scaffold, further immunohistochemistry analyses through multiple antibodies were performed to determine the phenotype of the cells. Indeed, the co-localization of Hoechst staining and the different antibodies permitted to reveal the nature of cells that have infiltrated the PLA- $b$-PHEMA scaffold. We found that among the cells that invaded the hydrogel, several were Ibal and vWF positive ones (Figure 8G-H).

\section{Discussion}

Previous to hydrogel implantation we confirmed the copolymer non-toxicity through motoneurons culture and its porosity by electron microscopy. Moreover, the kinetic of degradation of the biomaterial indicated a mass loss as soon as 55 days after being plunged into the degradation medium. The hydrogel was then implanted into $\mathrm{T}_{10}$ hemisected rats and functional recovery was assessed during 8 weeks through 2 complementary behavioral tests. Contrary to non-treated rats, significant improvement using the BBB locomotor test was observed in the implanted group at the $8^{\text {th }}$ week post-surgery. However, the implantation of the hydrogel did not induce benefit effect on the weight-bearing distribution. Finally, histological measurements confirmed axonal regeneration and tissue formation through the porous three-dimensional structure of the PLA- $b$-PHEMA hydrogel.

\subsection{PLA-b-PHEMA hydrogel properties.}

The non-toxicity of the PLA- $b$-PHEMA hydrogel was assessed on spin-coated polymeric material films, a procedure classically described for evaluating cell behavior on any biomaterial $[19,27]$. The qualitative assessment of the survival and the neurite growth of 
embryonic motoneurons during a period of 7 days highlighted the fact that the copolymer did not present any toxic effect on motoneurons and allowed robust neurite growth.

Concerning the degradation properties of the PLA- $b$-PHEMA, samples exhibited mass loss in simulated physiological conditions (in phosphate buffer, $\mathrm{pH} 7.4$ and at $37^{\circ} \mathrm{C}$ ). More precisely, reminiscent of the PLA homopolymer, the copolymer degradation process was characteristic of bulk erosion that maintained a constant mass during the first 8 weeks. Previous studies on PLA-b-PHEA analog material showed that an in vitro degradation mechanism and behavior were very similar to the one observed in vivo. Therefore, it can be reasonably assumed in the present study that the in vivo degradation of our PLA- $b$-PHEMA is mainly hydrolytical and close to the observed in vitro degradation [19]. The PLA- $b$-PHEMA material exhibited a bulk degradation mechanism characterized by a first time period $(\sim 55$ days) during which there is no mass loss but only random scission of the esters of the PLA segments inside the material, followed by the leaching of the PLA-based degradation products in a second phase ( $\sim 60 \%$ mass loss). The impact of the degradation process on spinal cord repair has been very few studied yet. Still, similar bulk eroding polymer scaffolds based on PLA or even PLA-PEG have brought promising results $[10,14,28]$. Moreover, the progressive formation of small oligolactide residues further increases softness and available space for penetration of axons inside the material.

Interestingly, our adapted freeze-extraction technique, consisting in the use of water as an extracting solvent, allowed the formation of the scaffold exempt of toxic and non-ecofriendly organic solvents [20]. Our hydrogel scaffold encompassed nanometer-sized pores as measured by electron microscopy. Moreover, we could reasonably assume that our hydrogel also included interconnected larger pores $(60-150 \mu \mathrm{m})$ such as the ones of the scaffolds previously prepared by the freeze-extraction method [20]. The multimodal pore size distribution of the PLA- $b$-PHEMA hydrogel is propitious for the free transport of molecules and the migration cells [29]. Therefore, this copolymer can be safely tested in vivo. 


\subsection{Functional assessment.}

As soon as the first week post-surgery, the decline in BBB score of the hemisected rats (from both $\mathrm{TH}$ and $\mathrm{TH}+$ Copolymer groups) was in accordance with previous studies, indicating important locomotor deficits after spinal cord hemisection (Figure 5) [28]. Although early behavioral improvement was noticed as soon as the $2^{\text {nd }}$ week post-surgery in all hemisected rats as formerly observed, hydrogel-implanted ones displayed a greater locomotor recovery at the $8^{\text {th }}$ week post-surgery [30]. Similar behavioral improvements were observed after lateral spinal cord hemisection followed by the well-known PHPMA implantation [23]. Likewise, better locomotor recovery through the BBB test was found after a dorsal hemisection followed by implantation of a radically crosslinked PEG hydrogel through lactide acrylate end groups [24]. However, contrary to our PLA- $b$-PHEMA copolymer, this partially degradable PEG hydrogel does not exhibit functional groups (such as hydroxyl ones) for further covalent immobilization of bioactive molecules.

An alteration in the weight-bearing distribution was observed in all hemisected animals, with or without hydrogel implantation (Figure 6 and 7). More precisely, the ipsilateral hindlimb to the spinal cord lesion supported less body weight than control rats. It is noteworthy to indicate that the lack of balance was not compensated by the contralateral hindlimb. However, the postural adaptation was characterized by a superior pressure exerted by the forelimbs. These data reinforced the ones previously obtained in our laboratory [22]. The complete recovery of the affected hindlimb weight-bearing capacities in the implanted rats appeared after a slightly longer delay than the non-treated ones.

The data obtained through the BBB and the DWB tests exhibited some differences that could be due to the fact that 1) the BBB test simplified the notion of weight-bearing support as absent, occasional ( $\geq 5 \%$ to $\leq 50 \%$ ), frequent $(>50 \%$ to $94 \%$ ) or consistent $(\geq 95 \%$ ) whereas the DWB test directly allowed quantification of the body-weight distribution and 2) the BBB estimated the weight-bearing support during locomotion while the DWB quantified the weight-bearing distribution during the static condition. 


\subsection{Spinal cord histology.}

This study confirmed that the implanted porous scaffold made of PLA- $b$-PHEMA copolymer remained into the lesional cavity of the hemisected spinal cord and appeared to be a suitable environment for tissue regeneration. Indeed, neurofilament fibers of regenerating axons were observed at the host tissue/hydrogel interface and into the biomaterial scaffold (Figure 8). Although those axonal fibers were noticed into the entire scaffold, further experiments with retrograde tracers need to be performed in order to specifically identify their pathway and their ability to bridge the spinal cord [31]. We speculate that a longer time delay could permit axonal outgrowth across the scaffold as it degrades.

As regards the GFAP staining, no astrocytic processes were observed into the implanted biomaterial. However, the effect of glial reactivity is still debated. Although glial scar is generally claimed to hamper axonal regrowth, it is also occasionally viewed as a necessary step for axonal regeneration [32]. Our results were in accordance to a previous study that reported an absence of GFAP signal into a PHEMA implant after 28 days postimplantation [33]. However, our findings contrast with another study that mentioned the invasion of the implant by astrocytic processes at 3 months post-surgery [34]. Those differences may be linked to the disparate experiment time period between the SCI and the animal sacrifice.

By performing additional immunohistochemistry analyses, we determined the phenotype of many nuclei that invaded the PLA- $b$-PHEMA scaffold. More precisely, the presence of both microglia and macrophages indicated a persistent inflammatory reaction 8 weeks post-surgery. Furthermore, the detection of vWF positive cells revealed the infiltration of some endothelial cells into the scaffold that might be the premise of angiogenesis, which was observed in a non-degradable PHPMA scaffold 3 months after implantation [34]. 


\section{Conclusions}

Our study showed that the prepared PLA- $b$-PHEMA block copolymer exhibits mandatory characteristics to be used as a CNS scaffold. Indeed, this block copolymer is a non-toxic platform that promotes neuron adhesion and neurite growth. Interestingly, after preparation of the porous scaffold through freeze-extraction method, the implantation of the copolymer led to locomotor improvements. This behavioral improvement may be supported by axonal regeneration into the hydrogel.

In addition, with this material, further relevant developments can be envisioned such as, controlled release of bioactive molecules (i.e. anti-inflammatory, neurotrophic factors) through the degradability and/or $\mathrm{OH}$ derivatization of the polymer. Moreover, the implementation of pore alignment and the evaluation on longer time period may emphasize axonal regeneration and functional recovery.

\section{Acknowledgments}

This work was supported by public [Aix-Marseille Université (AMU), Centre National de la Recherche Scientifique (CNRS) and Agence Nationale de la Recherche (ANR TRAUMATISM 09-BLAN-0295-02)] and private grants [Association Libre d'Aide à Recherche sur la Moelle Epinière (ALARME), Demain Debout and Combattre la Paralysie Associations]. This work was also supported by Association Méditerranéenne pour le Développement des Transplantations (AMDT). We are grateful to Jean-Louis CLEMENT for his help in the preparation of the porous scaffold and to Thelma COYLE for her help in Matlab data analysis. We are grateful to Alain DONNEAUD for technical support. 


\section{References}

[1] Thuret S, Moon LD, Gage FH. Therapeutic interventions after spinal cord injury. Nat Rev Neurosci 2006;7:628-43.

[2] Hejcl A, Sedy J, Kapcalova M, Toro DA, Amemori T, Lesny P, et al. HPMA-RGD hydrogels seeded with mesenchymal stem cells improve functional outcome in chronic spinal cord injury. Stem cells Dev 2010;19:1535-46.

[3] Hopkins AM, De Laporte L, Tortelli F, Spedden E, Staii C, Atherton TJ, et al. Silk hydrogels as soft substrates for neural tissue engineering. Adv Funct Mater 2013;23:5140-49.

[4] Hurtado A, Moon LD, Maquet V, Blits B, Jerome R, Oudega M. Poly (D,L-lactic acid) macroporous guidance scaffolds seeded with schwann cells genetically modified to secrete a bi-functional neurotrophin implanted in the completely transected adult rat thoracic spinal cord. Biomaterials 2006;27:430-42.

[5] Woerly S, Doan VD, Sosa N, de Vellis J, Espinosa-Jeffrey A. Prevention of gliotic scar formation by Neurogel allows partial endogenous repair of transected cat spinal cord. J Neurosci Res 2004;75:262-72.

[6] Nomura H, Tator $\mathrm{CH}$, Shoichet MS. Bioengineered strategies for spinal cord repair. J Neurotrauma 2006;23:496-507.

[7] Straley KS, Foo CW, Heilshorn SC. Biomaterial design strategies for the treatment of spinal cord injuries. J Neurotrauma 2010;27:1-19.

[8] von Recum HA, Cleek RL, Eskin SG, Mikos AG. Degradation of polydispersed poly(Llactic acid) to modulate lactic acid release. Biomaterials 1995;16:441-7.

[9] Zhong Y, Bellamkonda RV. Biomaterials for the central nervous system. J R Soc Interface 2008;5:957-75.

[10] Gautier SE, Oudega M, Fragoso M, Chapon P, Plant GW, Bunge MB, et al. Poly(alphahydroxyacids) for application in the spinal cord: resorbability and biocompatibility with adult rat Schwann cells and spinal cord. J Biomed Mater Res 1998;42:642-54.

[11] Oudega M, Gautier SE, Chapon P, Fragoso M, Bates ML, Parel JM, et al. Axonal regeneration into Schwann cell grafts within resorbable poly(alpha-hydroxyacid) guidance channels in the adult rat spinal cord. Biomaterials 2001;22:1125-36.

[12] Patist CM, Mulder MB, Gautier SE, Maquet V, Jerome R, Oudega M. Freeze-dried poly(D,L-lactic acid) macroporous guidance scaffolds impregnated with brain-derived neurotrophic factor in the transected adult rat thoracic spinal cord. Biomaterials 2004;25:1569-82.

[13] Pego AP, Poot AA, Grijpma DW, Feijen J. Biodegradable elastomeric scaffolds for soft tissue engineering. J Control Release 2003;87:69-79.

[14] Maquet V, Martin D, Scholtes F, Franzen R, Schoenen J, Moonen G, et al. Poly(D,Llactide) foams modified by poly(ethylene oxide)-block-poly(D,L-lactide) copolymers and aFGF: in vitro and in vivo evaluation for spinal cord regeneration. Biomaterials 2001;22:113746.

[15] Bakshi A, Fisher O, Dagci T, Himes BT, Fischer I, Lowman A. Mechanically engineered hydrogel scaffolds for axonal growth and angiogenesis after transplantation in spinal cord injury. J Neurosurg Spine 2004;1:322-9.

[16] Hejcl A, Lesny P, Pradny M, Michalek J, Jendelova P, Stulik J, et al. Biocompatible hydrogels in spinal cord injury repair. Physiol Res 2008;57 Suppl 3:S121-32.

[17] Vijayasekaran S, Chirila TV, Robertson TA, Lou X, Fitton JH, Hicks CR, et al. Calcification of poly(2-hydroxyethyl methacrylate) hydrogel sponges implanted in the rabbit cornea: a 3-month study. J Biomater Sci Polym Ed 2000;11:599-615.

[18] Clement B, Trimaille T, Alluin O, Gigmes D, Mabrouk K, Feron F, et al. Convenient access to biocompatible block copolymers from SG1-based aliphatic polyester macroalkoxyamines. Biomacromolecules 2009;10:1436-45.

[19] Clement B, Decherchi P, Feron F, Bertin D, Gigmes D, Trimaille T, et al. Poly(D,Llactide)-block-poly(2-hydroxyethyl acrylate) block copolymers as potential biomaterials for 
peripheral nerve repair: in vitro and in vivo degradation studies. Macromol Biosci 2011;11:1175-84.

[20] Ho MH, Kuo PY, Hsieh HJ, Hsien TY, Hou LT, Lai JY, et al. Preparation of porous scaffolds by using freeze-extraction and freeze-gelation methods. Biomaterials 2004;25:12938.

[21] Basso DM, Beattie MS, Bresnahan JC. A sensitive and reliable locomotor rating scale for open field testing in rats. J Neurotrauma 1995;12:1-21.

[22] Pertici V, Pin-Barre C, Felix MS, Laurin J, Brisswalter J, Decherchi P. A new method to assess weight-bearing distribution after central nervous system lesions in rats. Behav Brain Res 2014;259:78-84.

[23] Pertici V, Amendola J, Laurin J, Gigmes D, Madaschi L, Carelli S, et al. The use of poly(N-[2-hydroxypropyl]-methacrylamide) hydrogel to repair a T10 spinal cord hemisection in rat: a behavioural, electrophysiological and anatomical examination. ASN Neuro 2013;5:149-66.

[24] Piantino J, Burdick JA, Goldberg D, Langer R, Benowitz LI. An injectable, biodegradable hydrogel for trophic factor delivery enhances axonal rewiring and improves performance after spinal cord injury. Exp Neurol 2006;201:359-67.

[25] Henderson CE, Bloch-Gallego E, Camu W. Purified embryonic motoneurons. In: Cohen J, Wilkin GP, editors. Neural cell culture: a practical approach: Oxford University Press; 1995.

[26] Arce V, Garces A, de Bovis B, Filippi P, Henderson C, Pettmann B, et al. Cardiotrophin1 requires LIFRbeta to promote survival of mouse motoneurons purified by a novel technique. J Neurosci Res 1999;55:119-26.

[27] Karp JM, Shoichet MS, Davies JE. Bone formation on two-dimensional poly(DL-lactideco-glycolide) (PLGA) films and three-dimensional PLGA tissue engineering scaffolds in vitro. J Biomed Mater Res A 2003;64:388-96.

[28] Teng YD, Lavik EB, Qu X, Park KI, Ourednik J, Zurakowski D, et al. Functional recovery following traumatic spinal cord injury mediated by a unique polymer scaffold seeded with neural stem cells. Proc Natl Acad Sci U S A 2002;99:3024-9.

[29] Woerly S. Restorative surgery of the central nervous system by means of tissue engineering using Neurogel implants. Neurosurg Rev 2000;23:59-77.

[30] Cloud BA, Ball BG, Chen BK, Knight AM, Hakim JS, Ortiz AM, et al. Hemisection spinal cord injury in rat: the value of intraoperative somatosensory evoked potential monitoring. J Neurosci Methods 2012;211:179-84.

[31] Chen BK, Knight AM, de Ruiter GC, Spinner RJ, Yaszemski MJ, Currier BL, et al. Axon regeneration through scaffold into distal spinal cord after transection. J Neurotrauma 2009;26:1759-71.

[32] Shibuya S, Yamamoto T, Itano T. Glial and axonal regeneration following spinal cord injury. Cell Adh Migr 2009;3:99-106.

[33] Li HY, Fuhrmann T, Zhou Y, Dalton PD. Host reaction to poly(2-hydroxyethyl methacrylate) scaffolds in a small spinal cord injury model. J Mater Sci Mater Med 2013;24:2001-11.

[34] Woerly S, Pinet E, De Robertis L, Bousmina M, Laroche G, Roitback T, et al. Heterogeneous PHPMA hydrogels for tissue repair and axonal regeneration in the injured spinal cord. J Biomater Sci Polym Ed 1998;9:681-711. 


\section{Figures}

Figure 1: PLA-b-PHEMA characteristic. A. Synthesis pathway of the PLA- $b$-PHEMA block copolymer. B. ${ }^{1}$ H NMR spectrum (in DMSO-d6) of the PLA- $b$-PHEMA block copolymer after purification of the block copolymer by twice precipitation in diethyl ether. The molar mass of the PHEMA block was calculated based on the known molar mass of the PLA block.

Figure 2: Degradation of PLA-b-PHEMA block copolymer as a function of time. A. PLA- $b$ PHEMA block copolymer's mass loss (expressed as a percentage of its initial mass); B. Structural analysis by ${ }^{1} \mathrm{H}$ NMR analysis of the copolymer material versus time, showing the formation of oligolactic acid residues and their subsequent elimination.

Figure 3: Evaluation of toxicity of the PLA-b-PHEMA block copolymer through motor neuron culture. A-C. Polyornithine/laminin, PLA-b-PHEMA and THF coverslips at one day after cell culture, respectively. D-E. Polyornithine/laminin and PLA- $b$-PHEMA coverslips at 7 days after cell culture, respectively. The black and the white arrows highlight the growing axon. (Scale bar: $30 \mu \mathrm{m}$ ).

Figure 4: Porosity of the PLA-b-PHEMA scaffold by electron microscopy. (Scale bar: $2 \mu \mathrm{m}$ )

Figure 5: $B B B$ locomotor rating scale. Significant differences in the $\mathrm{BBB}$ scores are indicated by $*$ (TH pre- vs. TH post- $),+(\mathrm{TH}+\mathrm{Copolymer}$ pre- $v s$. TH+Copolymer post-), $\delta$ (Control and SHAM groups vs. TH and $\mathrm{TH}+\mathrm{Copolymer}$ groups) and $\Omega$ (TH group vs. $\mathrm{TH}+$ Copolymer group). (One symbol $\mathrm{p}<0.05$ and 3 symbols $\mathrm{p}<0.001$ ).

Figure 6: Pressure exerted by each paw after thoracic hemisection. The pressure exerted by each paw is expressed as a percentage of the total weight. A. Pressure of the left forepaw. B. Pressure of the right forepaw. C. Pressure of the left hindpaw. D. Pressure of the right hindpaw. Significant differences in the pressure exerted by each paw are indicated by * (TH pre- vs. TH post-), + (TH+Copolymer pre- vs. TH+Copolymer post-) and $\Omega$ (TH group $v s$. $\mathrm{TH}+$ Copolymer group). (One symbol $\mathrm{p}<0.05 ; 2$ symbols $\mathrm{p}<0.01$ and 3 symbols $\mathrm{p}<0.001$ ).

Figure 7: Pressure ratios after thoracic hemisection. A. The sum of pressure exerted by the forepaws $(\mathrm{F})$ normalized by the sum of pressure exerted by the hindpaws $(\mathrm{H})$. B. The pressure exerted by the left forepaw (LF) normalized by the pressure exerted by the right forepaw (RF). C. The pressure exerted by the left hindpaw (LH) normalized by the pressure exerted by the right hindpaw (RH). Significant differences in the pressure ratios are indicated by * (TH pre- $v s$. TH post- $),+(\mathrm{TH}+$ Copolymer pre- $v s$. TH + Copolymer post- $)$ and $\Omega$ (TH group $v s$. $\mathrm{TH}+$ Copolymer group). (One symbol $\mathrm{p}<0.05 ; 2$ symbols $\mathrm{p}<0.01$ and 3 symbols $\mathrm{p}<0.001$ ).

Figure 8: Histological analyses. A-B. Hoechst staining of hydrogel-implanted spinal cords in coronal and longitudinal views, respectively. C. Hoechst (blue) costaining with NF-H (red) and GFAP (green) antibodies of a hydrogel-implanted spinal cord. While numerous Hoechst cells invaded the hydrogel scaffold, no GFAP positive cells were found within the biomaterial. Moreover, NF-H positive fibers were observed in the hydrogel (Scale bar: 200 $\mu \mathrm{m})$. D-F. Magnification of the squares localized in C. G-H. Hoechst costaining with Iba1 (red) and vWF (green) antibodies, respectively (Scale bar: $20 \mu \mathrm{m}$ ). 